Z. Wahrscheinlichkeitstheorie verw. Gebiete 31, 161 (1975)

(C) by Springer-Verlag 1975

\title{
Erratum
}

\section{Random Ergodic Theorem with Weighted Averages}

\author{
T. Yoshimoto \\ Z. Wahrscheinlichkeitstheorie verw. Gebiete 30, 149-165 (1974)
}

In Sections 2 and 3 it is not correct to consider $\mu^{*}$ as the product measure of a family $\left\{\mu_{n}\right\}$ of arbitrary probability measures defined on the given measurable space $\left(\Phi^{*}, \mathscr{F}^{*}\right)$. Instead let $\mu^{*}$ be a probability measure defined on $\mathscr{F}^{*}$ by the requirement that the coordinate sequence $\left\{\xi_{n}\left(\varphi^{*}\right)\right\} \quad\left(\xi_{n}\left(\varphi^{*}\right)=\varphi_{n}\right.$ if $\left.\varphi^{*}=\left\{\varphi_{n}\right\}\right)$ should be a (strongly) stationary process, and let $\sigma$ be the shift transformation on $\Phi^{*}$. Then, since $\sigma$ preserves the measure $\mu^{*}$, the dynamical system $\left(\Phi^{*}, \mathscr{F}^{*}, \mu^{*}, \sigma\right)$ is enough for our purpose.

Remark 10 should be omitted. (If $\sigma$ is a Bernoulli shift then this remark remains true.)

\author{
Takeshi Yoshimoto \\ Department of Mathematics \\ Faculty of Engineering \\ Toyo University \\ Kawagoe, Saitama, Japan
}

